\title{
A Phase Diagram for Wrapping
}

\author{
Researchers find the conditions for when a cell membrane will wrap \\ around a plastic bead, providing insight into how living things interact \\ with viruses, microplastics, and other objects.
}

By Katherine Wright

W hen a cell gets hungry, it wraps a nearby food particle with a section of its outer membrane, creating a food-containing pouch that gets "swallowed" whole. Cells drink fluids and ingest viruses in the same way, making it important to understand the conditions required for the wrapping process to take place. Now Eric Dufresne of the Swiss Federal Institute of Technology (ETH) in Zurich and his colleagues have experimentally mapped out these conditions for a plastic bead and a model membrane [1]. The results could help researchers understand when microplastics will enter cells.

For their experiments, the team placed micrometer-diameter plastic beads in a solution containing artificial cells, each of which consisted of a nanometer-thick spherical membrane. The composition of the solution acted as a knob that allowed the team to indirectly tune the strength of the adhesion interaction between a bead and the membrane, something that controls how easily a membrane "catches" a bead. The team carried out experiments for various adhesion strengths and monitored what happened with a microscope.

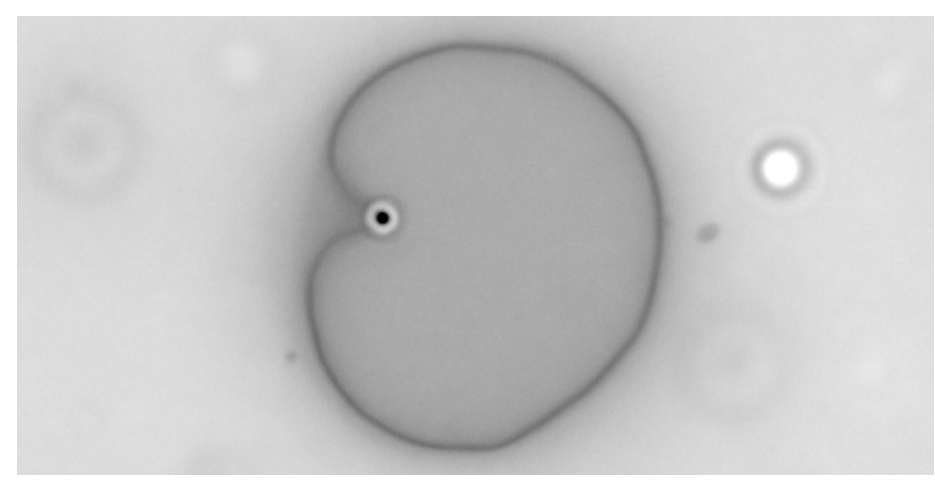

Credit: H. T. Spanke et al., Phys. Rev. Lett. (2020)
Dufresne and colleagues observed three different behavior regimes. At low particle-membrane adhesion, no wrapping occurred. At intermediate adhesion, wrapping sometimes occurred, but only if the particle was physically pushed into the membrane with a laser. For high adhesion, wrapping always occurred, and this process was spontaneous.

The team speculates that living systems take advantage of all three interaction regimes. But they note that their idealized experimental system may have limited applicability to real cells. Thus, they hope that the methods that they developed will be applied to more complex systems that more closely mimic those found in living things.

Katherine Wright is a Senior Editor for Physics.

\section{REFERENCES}

1. H. T. Spanke et al., "Wrapping of microparticles by floppy lipid vesicles," Phys. Rev. Lett. 125, 198102 (2020). 